\title{
Total Muon Capture Rates and Mean Neutrino Energy.
}

P. Christillin, A. Dellafiore and M. Rosa-Clot

Scuola Normale Superiore - Pisa, Italia

Istituto Nazionale di Fisica Nucleare - Sezione di Pisa, Italia

(Nuovo Cimento, 34 A, $272(1976))$

Some trivial misprints appear in sect. 3 .

Equation (25) should read

$$
\left.\cdots+\frac{1}{128} \frac{1}{x^{3}} \gamma_{-}^{3}\right\}
$$

Equation (36)

$$
R=\frac{1}{Z} \int \nu^{2} S \mathrm{~d} n_{Z} \mathrm{~d} \nu \delta(\mu-\varepsilon-v-E)
$$

Equation (37)

$$
R=\int R(E) \mathrm{d} E=\frac{1}{Z} \int_{a}^{b} \nu^{2} S_{1} \frac{M^{*}}{\nu} \mathrm{d} E+\frac{1}{Z} \int_{b}^{c} v^{2} S_{2} \frac{M^{*}}{\nu} \mathrm{d} E .
$$

\section{Isotope Effect in Total Muon Capture Rates on Nuclei and Isotensor Effective Potential.}

P. Christillin and M. Rosa-Clot

Scuola Normale Superiore - Pisa, Italia

Istituto Nazionale di Fisica Nucleare - Sezione di Pisa, Italia

A. Dellafiore

Department of Theoretical Physics, Oxford University - Oxford, Great Britain (Nuovo Cimento, 34 A, 296 (1976))

Equation (7) should read

$$
\cdots+\frac{k x}{1-k \alpha} \frac{2 Z}{A(A+x)} .
$$

